\title{
Optimizing stringency for expression microarrays
}

\author{
James E. Korkola, Anne L.H. Estep, Sunanda Pejavar, Sandy DeVries, \\ Ronald Jensen, and Frederic M. Waldman
}

BioTechniques 35:828-835 (October 2003)

\begin{abstract}
While several studies have reported methods to optimize expression microarray protocols, none have dealt directly with hybridization wash stringency. We designed a series of experiments to determine the optimal stringency conditions for microarray experiments, using reproducibility and magnitudes of $\log _{2}$ (test/reference) ratio values as measures of quality. Low-stringency wash conditions of cell line hybridizations led to nonspecific binding, resulting in increased intensities, decreased magnitude of ratios, and poor reproducibility. Relatively high-stringency wash conditions were found to give the best reproducibility and large magnitude ratio changes, although increasing the stringency beyond this point led to lower magnitude ratios and poorer reproducibility. The expression levels of the ERBB2 oncogene in the BT474 versus MCF7 cell lines showed that high-stringency wash conditions gave the best agreement with real-time quantitative PCR, although the magnitude of the changes by microarray was smaller than for real-time quantitative PCR. Analysis of a series of cell lines washed at the optimized stringency indicated that the rank order of relative expression levels for ERBB2 microarray clones agreed well with the rank order of ERBB2 levels, as measured by quantitative PCR. These results indicate that the optimization of stringency conditions will improve microarray reproducibility and give more representative expression values.
\end{abstract}

\section{INTRODUCTION}

The ability to survey thousands of genes in a single sample and to recognize complex patterns in related samples that predict parameters, such as gene function (1), clinical response (2), or cell cycle phases (3), has made the microarray an important discovery tool. A number of studies have reported methods for optimizing microarray protocols. These include reports on the preparation of arrays and labeling of probe (4), amplification of RNA for use in microarrays (5), and analysis of the resulting data (6). Although several reports have briefly discussed stringency $(7,8)$, no reports have directly examined stringency effects on cDNA microarray results. The optimization of stringency conditions should improve the results from array experiments. If conditions are too stringent, then specific signal will be washed from the microarray, leading to the loss of signal and therefore lower signal-to-noise ratios. Conversely, low-stringency conditions will lead to nonspecific probe binding and masking of specific signal.

We designed an experiment to test the effects of wash stringency on microarray results. A range of wash conditions with different stringencies was tested using four breast cell lines and a reference pool hybridized against itself. High-stringency wash conditions gave the best results, with better reproducibility and ratios with larger magnitude changes than lower stringency washes. Analysis of the ERBB2 gene indicated that at the best stringency condition, there was good agreement between real-time quantitative PCR and microarray results. These results show that high-stringency washes improve expression microarray reproducibility and give more representative expression values.

\section{MATERIALS AND METHODS}

\section{Samples}

Breast cancer cell lines BT474, MCF7, T47D, and MDA231 (ATCC,
Manassas, VA, USA) were obtained from the University of California San Francisco (UCSF) Cell Culture Facility (San Francisco, CA, USA). RNA was isolated from subconfluent flasks using TRIzOL ${ }^{\circledR}$ Reagent (Invitrogen, Carlsbad, CA, USA), according to the manufacturer's instructions. RNA quantity and quality were determined by using ultraviolet absorption at $260 \mathrm{~nm}$ and by running 100 ng RNA on denaturing agarose gels. The RNA was then DNase-digested using DNA-free ${ }^{\mathrm{TM}}$ (Ambion, Austin, TX, USA). A reference cell line RNA pool for the microarray hybridizations was prepared using TRIzOL and consisted of equal amounts of RNA from the following cell lines: MCF7 (breast adenocarcinoma); HepG2 (hepatocellular carcinoma); MOLT-4 (T cell leukemia); NTERA2 (teratoma); SW872 (liposarcoma); WM115 (melanoma); OVCAR3 (ovarian carcinoma); RPMI8226 (mutiple myeloma); Colo205 (colon adenocarcinoma); Hs578T (breast carcinosarcoma); and HL60 (acute promyelocytic leukemia) (ATCC). 
ng total RNA were reverse-transcribed using Moloney murine leukemia virus (MMLV) reverse transcriptase and random hexamer primers (both from Invitrogen), according to standard protocols (12). The samples were then subjected to real-time PCR analysis using an ABI PRISM ${ }^{\circledR} 7700$ real-time thermal cycler (Applied Biosystems) at the UCSF Comprehensive Cancer Center Genome Analysis Core. The probes used were ERBB2, $\beta$-glucuronidase ( $\beta$-Gus), and GAPDH.

\section{RESULTS}

\section{Stringency Effects on Intensity}

Table 2 shows the average intensity for test and reference channels, overall standard deviation of the $\log _{2}$ (test/ reference) ratios, and the number of spots included (as a measure of spot intensity, related to nonspecific probe binding for low-expressing genes) for the samples at different stringency conditions. The standard deviation is a reflection of the heterogeneity of expression levels on the array. For every sample, the overall standard deviation of the $\log _{2}$ (test/reference) ratios was lowest at the lowest stringency, increased for the next two stringency levels, and decreased for the highest stringency level. The total number of spots included in each hybridization tended to decrease from low stringency to high stringency. The average intensities were significantly higher in the samples washed at the lowest stringency $(P<0.01)$. For the other wash conditions, the intensities did not differ from each another.

The average background levels for both $\mathrm{Cy} 5$ and $\mathrm{Cy} 3$ did not change with stringency. The average of the median background intensities for $\mathrm{Cy} 5$ across the five different cell lines were 204 $\left(20^{\circ} \mathrm{C}\right), 181\left(42^{\circ} \mathrm{C}\right), 203\left(55^{\circ} \mathrm{C}\right)$, and $189\left(65^{\circ} \mathrm{C}\right)$. For $\mathrm{Cy} 3$, the average background intensities were $308\left(20^{\circ} \mathrm{C}\right), 314$ $\left(42^{\circ} \mathrm{C}\right), 321\left(55^{\circ} \mathrm{C}\right)$, and $322\left(65^{\circ} \mathrm{C}\right)$.

\section{Reproducibility and Ratio Magnitudes}

For each cell line, we selected clones that showed changes $\left(\log _{2}\right.$ ratios greater than 1 or less than -1 ) and

Table 2. Quality Measures of Expression Array Hybridizations

\begin{tabular}{|c|c|c|c|c|c|}
\hline Sample & $\begin{array}{c}\text { Condition } \\
\left({ }^{\circ} \mathrm{C}\right)\end{array}$ & Суза & Сy5 $5^{b}$ & $\begin{array}{c}\text { SD Test/ } \\
\text { Reference }^{\text {b }}\end{array}$ & $\begin{array}{c}\text { Spots }^{c} \\
(n)\end{array}$ \\
\hline \multirow[t]{4}{*}{ BT474 } & 20 & 2712 & 5036 & 0.436 & 5528 \\
\hline & 42 & 1150 & 1186 & 0.650 & 5658 \\
\hline & 55 & 1240 & 1251 & 0.701 & 5497 \\
\hline & 65 & 1026 & 1540 & 0.600 & 5512 \\
\hline \multirow[t]{4}{*}{ MCF7 } & 20 & 3690 & 8097 & 0.226 & 5571 \\
\hline & 42 & 754 & 1870 & 0.472 & 5666 \\
\hline & 55 & 638 & 1420 & 0.587 & 5537 \\
\hline & 65 & 875 & 1996 & 0.508 & 5535 \\
\hline \multirow[t]{4}{*}{ MDA231 } & 20 & 2535 & 4906 & 0.337 & 5591 \\
\hline & 42 & 864 & 1380 & 0.520 & 5632 \\
\hline & 55 & 1077 & 1343 & 0.600 & 5509 \\
\hline & 65 & 989 & 1568 & 0.550 & 5233 \\
\hline \multirow[t]{4}{*}{ T47D } & 20 & 1552 & 2100 & 0.425 & 5522 \\
\hline & 42 & 1240 & 1721 & 0.550 & 5681 \\
\hline & 55 & 1001 & 1274 & 0.686 & 5223 \\
\hline & 65 & 926 & 1417 & 0.555 & 5378 \\
\hline \multirow[t]{4}{*}{ Reference } & 20 & 2452 & 5079 & 0.187 & 5640 \\
\hline & 42 & 1109 & 1749 & 0.247 & 5521 \\
\hline & 55 & 810 & 1271 & 0.291 & 5053 \\
\hline & 65 & 877 & 1619 & 0.286 & 5359 \\
\hline \multicolumn{6}{|c|}{$\begin{array}{l}\text { SD, standard deviation. } \\
\text { aAverage of mean intensity (background-subtracted) for all spots included in } \\
\text { analysis. } \\
\text { bOverall standard deviation of all corrected } \log _{2}(\text { test/reference) ratios. } \\
\text { cTotal number of cDNAs included in analysis. }\end{array}$} \\
\hline
\end{tabular}

were represented multiple times on the arrays to define the reproducibility of array results. These clones were used to calculate the means and standard deviations. There were 42 clones that were present with multiple copies printed on the arrays that showed alterations in at least one cell line. There were 31 clones in BT474, 19 clones in MCF7, 25 in MDA231, and 19 in T47D that met these criteria. Table 3 shows the average standard deviations for each cell line and the reference pool at each stringency level. The average of the standard deviations of the $\log _{2}$ (test/ reference) ratios for the selected clones was lowest when the slides were washed at $20^{\circ}$, followed by $55^{\circ}, 42^{\circ}$, and $65^{\circ} \mathrm{C}$, respectively.

In addition to clones that were pres- ent multiple time that showed changes, we also examined all the clones that showed a $\log _{2}$ ratio change averaging greater than 1 or less than -1 in a cell line, regardless of how many copies were present. The ratio values for these clones were plotted for each cell line by stringency level, as shown in Figure 1 . The number of clones that fit this criterion varied by cell line, with BT474 having the most clones that were above 1 or below -1 , and MCF 7 having the fewest. A moving average was calculated to smooth the plot to make visualization easier (the ratio value for each clone was averaged with the two previous and two following ratio values). In each case, arrays washed at $55^{\circ} \mathrm{C}$ showed the largest magnitude changes for clones that had low levels of expres- 
Table 3. Average Standard Deviations of Selected Clones at the Four Stringency Levels

\begin{tabular}{|lcccc|}
\hline & \multicolumn{4}{c|}{ Wash No. 1 Temperatures } \\
\cline { 2 - 5 } Sample $^{\mathbf{a}}$ & $\mathbf{2 0}^{\circ} \mathbf{C}$ & $\mathbf{4 2}^{\circ} \mathbf{C}$ & $\mathbf{5 5}^{\circ} \mathbf{C}$ & $\mathbf{6 5}^{\circ} \mathbf{C}$ \\
\hline BT474 $(n=31)$ & $0.278^{\mathrm{b}}$ & 0.385 & 0.261 & 0.305 \\
MCF7 $(n=19)$ & 0.235 & 0.313 & 0.353 & 0.338 \\
MDA231 $(n=25)$ & 0.296 & 0.301 & 0.313 & 0.418 \\
T47D $(n=19)$ & 0.192 & 0.347 & 0.284 & 0.335 \\
reference $(n=42)$ & 0.141 & 0.165 & 0.117 & 0.172 \\
Average & 0.228 & 0.302 & 0.266 & 0.314 \\
a $n$ represents the total number of clones that showed changes and were repre- \\
sented multiple times on the arrays for each cell line. \\
bValues represent the average of the standard deviations for each set of replicate \\
\multicolumn{2}{l}{ clones for a given cell line at the different wash temperatures. } \\
\hline
\end{tabular}

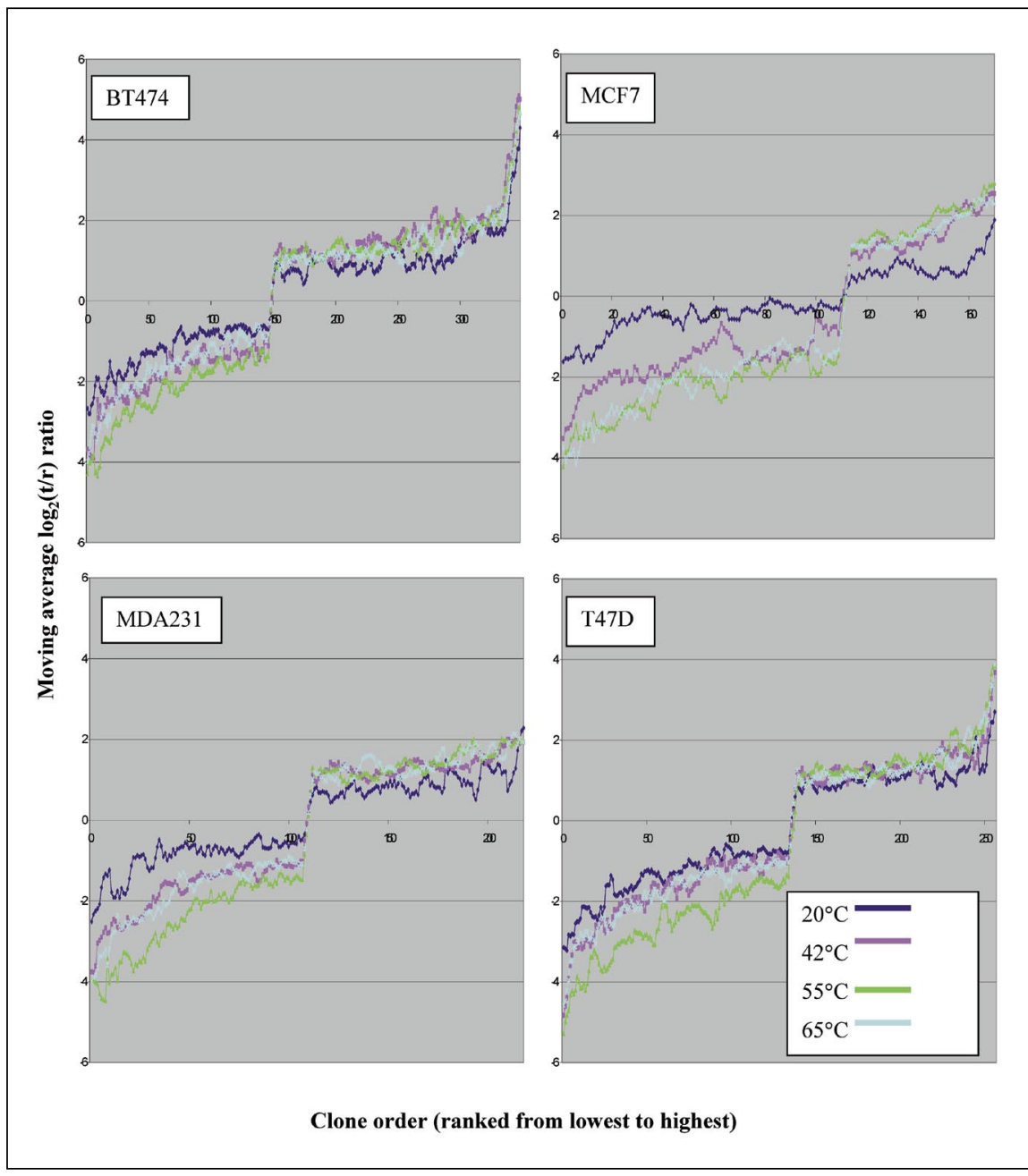

Figure 1. Moving average $\log _{2}$ (test/reference) ratios of all clones that showed alterations for each stringency level. To smooth the curve, a moving average was calculated by averaging the ratio for a given clone with the two previous and two following ratios. The $\mathrm{x}$-axis represents the clones ordered from the lowest average expression to highest average expression. 
sion. For clones showing high levels of expression, there did not appear to be any differences between washes at $42^{\circ}$, $55^{\circ}$, or $65^{\circ} \mathrm{C}$. For both low- and highexpressing clones, the arrays washed at $20^{\circ} \mathrm{C}$ had the lowest magnitude ratios.

\section{Stringency Effects on Clustering}

The four sets of cell line and reference samples were clustered using Eisen's Treeview/Cluster software package. The data were centered to a $\log _{2}$ ratio of zero. No further normalizations were performed. Figure 2 shows the clustering of samples. Note that for MCF7, the sample washed at $20^{\circ} \mathrm{C}$ did not cluster with the MCF7 samples washed at higher stringencies. Instead, this sample was higher up the cluster dendrogram, clustering with the MCF7, BT474, and MDA231 samples. The BT474 and T47D samples did not show any significant effect of stringency on clustering, but the sample washed at $20^{\circ} \mathrm{C}$ for MDA231 was the most weakly clustering sample.

\section{ERBB2 Array Data versus Real-Time Quantitative PCR}

The BT474 breast cancer cell line is known to overexpress the ERBB2 oncogene, while the MCF7 breast cancer cell line shows normal levels of expression. TaqMan measures ERBB2 expression $125 \times$ higher in BT474 than in MCF7. The levels of ERBB2 in BT474 compared to MCF7 from microarrays were calculated for each stringency level. The $20^{\circ} \mathrm{C}$ wash showed the lowest ratio (17.9:1), followed by the $42^{\circ} \mathrm{C}$ wash $(22.6: 1), 65^{\circ} \mathrm{C}$ wash $(23.0: 1)$, and $55^{\circ} \mathrm{C}$ wash (32.2:1).

Washes at $55^{\circ} \mathrm{C}$ were determined to be optimal due to the best reproducibility and largest magnitude ratios. A series of hybridizations were done to compare hybridization results at this stringency level to real-time quantitative PCR data. Each of the 11 cell lines in the reference pool plus BT474 was hybridized versus the reference pool to arrays, which consisted of $10,368 \mathrm{cDNA}$ clones. The normalized relative levels of expression of $E R B B 2$ for two sequenceverified clones of this gene were compared to relative real-time quantitative PCR levels. Figure 3 shows the level of expression for the $E R B B 2$ gene (relative to HL60, which was defined as a level of 1 for both arrays and real-time quantitative PCR) across 11 separate cell lines. The data shown are the expression levels for the two separate cDNA clones and real-time quantitative PCR (normalized to either $\beta-G u s$ or $G A P D H)$. The cell lines were ordered from lowest to highest expression, and the order as measured by the expression array was the same for both cDNA clones. The order for real-time quantitative PCR quantitation differed depending on the control gene used for normalization, as can be seen in Figure 3. The order for the cDNA clones agreed well with the real-time quantitative PCR levels relative to either control gene, although the magnitude of expression differences was less by microarray analysis.

\section{DISCUSSION}

The wash stringency can have a profound effect on expression array hybridization results. Stringency levels need to be high enough to prevent nonspecific binding, but not so high that specific signal is removed by the wash. We designed a study to examine the effects of wash stringencies on microarray results, using the magnitude of changes, reproducibility, and clustering as measures of the quality of hybridization results.

Stringency can be altered by changing the salt concentration, adding formamide, or altering temperature. We chose to alter one of these parameters, keeping the others constant to more easily interpret the results. Thus, we varied the temperature as a means of testing different stringency levels during microarray washes.

The first measure of quality was the standard deviation of the $\log$ (test/ reference) ratios of the spotted cDNA clones. If the stringency is too low, then nonspecific binding will occur, leading to a lower overall average standard deviation of all the $\log$ (test/reference) ratios for an experiment and the inclusion of more spots. If stringency is too high, then specific signal will be washed off, which will decrease the signal intensity above background. This would likely result in poorer reproducibility, a smaller number of spots passing quality controls, and may decrease the magnitude of ratios. The ideal stringency should give a large standard deviation in a cell line versus reference hybridization (the largest standard deviation will give the largest spread of ratio values) with good reproducibility and the inclusion of the most spots. We found that the washes at $55^{\circ} \mathrm{C}\left(\Delta \mathrm{T}=38^{\circ} \mathrm{C}\right)$ gave the highest standard deviations in all the cell lines, indicating that it may be the best of the conditions tested. Washes at room temperature $\left(20^{\circ} \mathrm{C} ; \Delta \mathrm{T}=73^{\circ} \mathrm{C}\right)$ showed evidence of nonspecific binding, with standard deviations that were comparable to reference versus reference hybridizations for the MCF7 and T47D hybridizations. The two remaining stringency levels showed consistently lower standard deviations than the $55^{\circ} \mathrm{C}$ wash level, indicating that there may have been loss of specific signal and increase in noise for the highest stringency wash or nonspecific binding for the lower stringency wash. The interpretation of these results is that there is some nonspecific binding with a $42^{\circ} \mathrm{C}$ first wash $\left(\Delta \mathrm{T}=51^{\circ} \mathrm{C}\right)$ and some removal of specific binding with a $65^{\circ} \mathrm{C}$ first wash $\left(\Delta \mathrm{T}=28^{\circ} \mathrm{C}\right)$.

The average $\mathrm{Cy} 3$ and $\mathrm{Cy} 5$ intensities varied by stringency level. The slides with the lowest stringency showed the

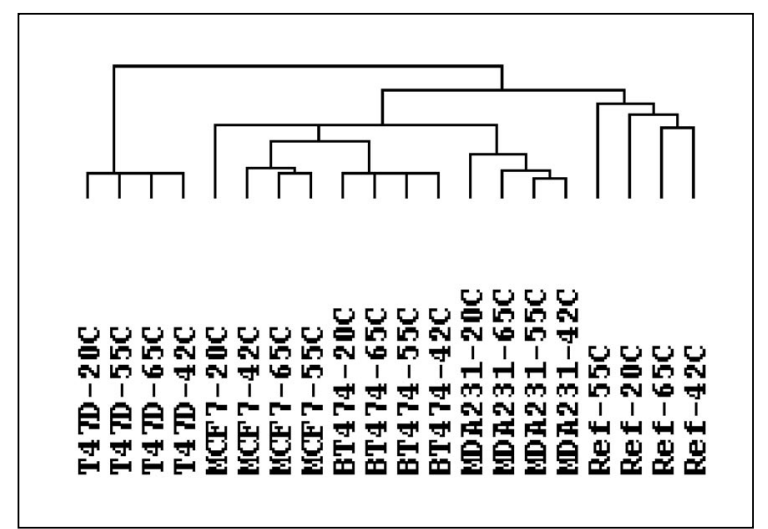

Figure 2. Hierarchical clustering of samples. Unsupervised clustering was performed for four cell lines and the reference sample washed under four different conditions, as indicated by the different temperatures. Note that the $20^{\circ} \mathrm{C}$ sample for MCF7 does not cluster with the higher stringency MCF7 samples. 
greatest intensity values, indicating nonspecific binding. There were no differences between the intensities for the slides washed at the other stringency levels. This was somewhat surprising, given that the $42^{\circ} \mathrm{C}$ wash exhibited characteristics of nonspecific binding, while the $65^{\circ} \mathrm{C}$ wash showed some signs of the removal of specific binding.

The background intensity did not change with increasing stringency but remained constant across all stringency levels. This result is not surprising because the same concentration of SDS was present in wash 1 for all stringency levels. SDS is a detergent that should prevent probes from sticking to the surface of the slide, but it has little effect on nucleic acid hybridization.

We expected that when three serial washes are used (as in this experiment), the most stringent condition would determine overall stringency. This was not the case in these experiments. As can be seen in Table 1, samples washed initially at either $20^{\circ}$ or $42^{\circ} \mathrm{C}$ had a minimum $\Delta \mathrm{T}$ of $51^{\circ}$ (third wash for $20^{\circ}$; first and third washes for $42^{\circ} \mathrm{C}$ ). However, the results from the experiments clearly indicate that the two wash conditions were not identical. This may reflect the time spent in the highest stringency wash (two 10-min washes for the samples initially washed at $42^{\circ} \mathrm{C}$, compared to one 10 -min wash for the sample initially washed at $20^{\circ} \mathrm{C}$ ).

To determine the stringency effects on the reproducibility and magnitude of the changes of the genes, a series of specific genes were chosen for quantitative analysis. These genes showed alterations in expression $\left(\log _{2}\right.$ test/reference ratio averaging greater than 1 or less than -1 across the stringency levels) in the specific cell line and were represented multiple times on the arrays. The standard deviation for each set of replicate genes was calculated, and an average standard deviation of all the clones was determined for each of the cell lines. Two of the four cell lines (BT474 and T47D) had the lowest standard deviations among replicates when washed at $55^{\circ} \mathrm{C}$. The remaining two cell lines (MCF7 and MDA231) had the lowest standard deviation among replicates when washed at $42^{\circ} \mathrm{C}$. The clones had the lowest standard deviation in the reference when the washes were performed at $55^{\circ} \mathrm{C}$. These results indicate that good reproducibility could be obtained when washes were performed at either $42^{\circ}$ or $55^{\circ}$, but that $55^{\circ} \mathrm{C}$ washes gave slightly

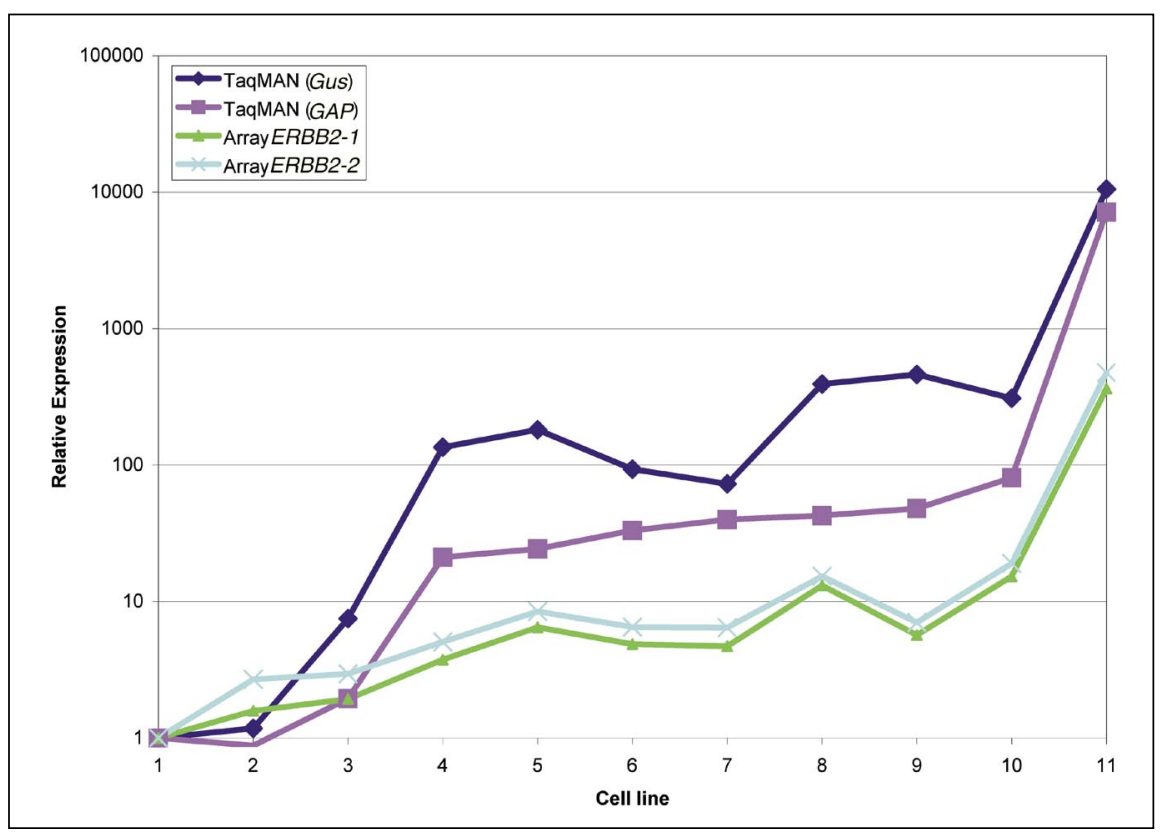

Figure 3. Relative expression levels for the $E R B B 2$ gene measured by microarray or real-time quantitative PCR analysis. All samples were washed at the $55^{\circ} \mathrm{C}$ stringency condition. Real-time quantitative PCR was normalized relative to either $G A P D H$ or $\beta$-glucuronidase ( $\beta$-Gus). Expression for all samples is relative to HL-60 (set to 1). The cell lines used are as follows: 1, HL60; 2, RPMI 8226; 3, MOLT-4; 4, Hs578t; 5, NTERA-2; 6, MCF7; 7, SW872; 8, HepG2; 9, OVCAR3; 10, WM115; 11, BT474. 
better reproducibility.

When the magnitude of the changes was examined for all the genes, the magnitude of the change was greatest when the washes were performed at $55^{\circ} \mathrm{C}$ for most clones (Figure 1). This was particularly true for clones that showed low levels of expression relative to the reference. For clones that showed high levels of expression relative to the reference, the result was not as clear. While $55^{\circ} \mathrm{C}$ washes clearly showed a larger magnitude in the ratio than washes at $20^{\circ} \mathrm{C}$, the differences between $42^{\circ}, 55^{\circ}$, and $65^{\circ} \mathrm{C}$ washes were minor. The differences between the high-expressing clones varied more than the low-expressing genes, with the sample with the largest magnitude ratio varying, depending on the clone as opposed to the wash stringency. There were also significant differences depending on which cell line was being examined, with MCF7 showing higher magnitude changes at $55^{\circ} \mathrm{C}$ than at the other high-stringency conditions for the high-expression clones. The other cell lines did not show such differences. The variability observed is likely due to the complex nature of the probe being hybridized. The stringency depends on factors such as the \%GC content and the length of the probes. For each $1.0^{\circ}-1.5^{\circ} \mathrm{C}$ drop in melting temperature, there is a $1 \%$ increase in mismatch allowed (10). Thus, for a probe-target hybrid that is $600 \mathrm{bp}$ in length, with a $\%$ GC content of $50 \%$, the $55^{\circ} \mathrm{C}$ wash condition would allow for an approximate $25 \%$ mismatch between the probe and target, while the $42^{\circ} \mathrm{C}$ wash would allow for a $34 \%$ mismatch. Because the length and \%GC are not fixed values for microarrays, the percentage of mismatches will vary for each probe-target hybrid. On average, $55^{\circ} \mathrm{C}$ washes give the best reproducibility and largest magnitude changes for genes that have low expression and the best reproducibility for high-expressing genes with comparable magnitude changes. The reason why the low-expressing genes show a consistent difference while high-expressing genes do not is unclear but may be due to different binding kinetics based on the Cy5 label compared to $\mathrm{Cy} 3$ label.

The clustering of the samples was done using Eisen's Treeview/Cluster software. Normalization was not done on these samples because they were not washed under the same conditions. The normalization of each sample is usually performed to ensure that the standard deviations of each sample are equivalent to correct for factors such as labeling efficiency and hybridization kinetics. In this case, normalization was not appropriate because stringency was expected to have a direct effect on the overall standard deviation, and this was one of the variables being tested. Furthermore, reference versus reference samples was included in the clustering, and normalization would be inappropri- 
ate for such samples. The clustering of non-normalized samples shows that the $20^{\circ} \mathrm{C}$ washed sample for MCF7 does not cluster with the higher stringency MCF7 samples. For MDA231, the $20^{\circ} \mathrm{C}$ wash was also the most weakly clustering sample, as shown by the distance on the clustering dendrogram.

Real-time quantitative PCR analysis was performed on the breast cell lines BT474 and MCF7 for the ERBB2 oncogene. This analysis showed that BT474 expressed approximately $125 \times$ as much $E R B B 2$ as MCF7. A comparison of the array-based BT474:MCF7 levels for the $E R B B 2$ gene showed that the $55^{\circ} \mathrm{C}$ wash came closest to this level. Real-time quantitative PCR analysis of the expression levels in a number of other cell lines for the $E R B B 2$ gene was performed for comparison to the relative levels, as determined by microarray experiments at the optimized $55^{\circ} \mathrm{C}$ wash stringency. Ranking of the cell lines by ERBB2 levels showed that the order was similar for real-time quantitative PCR compared to two separate microarray clones. The rank order for the two clones was identical, whereas the real-time quantitative PCR rank order varied depending on which housekeeping gene ( $\beta-G u s$ or GAPDH) was used for normalization. The levels of expression were less for microarrays than for real-time quantitative PCR, which is likely due to hybridization effects such as background, spot saturation, steric effects, and dye effects.

These results are limited to the cDNA platform and two-color hybridizations. Alternative approaches use oligonucleotide arrays and one- or twocolor analyses. Oligonucleotides have hybridization characteristics that are different from cDNAs because oligonucleotides have high specificity (unique sequence) but short lengths, which affects the hybridization and melting of double-stranded DNA. The results of this study are not directly applicable to oligonucleotide arrays, but stringency conditions with these arrays should also be optimized and validated.

We show the importance of optimizing stringency in microarray experiments. The stringency must be a balance between intensity and specificity, so that the signal is maximized while minimizing nonspecific probe binding. The results presented here indicate that high-strin- gency washes in microarray experiments are preferable to low-stringency washes. The best condition found in these experiments was higher than is typically used in cDNA microarray experiments, which raises the possibility that experiments performed at lower stringency may have missed some differentially expressed genes. Changing current protocols to optimize stringency would likely improve reproducibility. High-stringency washes result in higher magnitude ratios, better reproducibility in expression microarray experiments, and expression values that are more representative of true cellular mRNA quantities.

\section{ACKNOWLEDGMENTS}

The authors wish to thank Dr. J. DeRisi for use of his arrayer robot, Jane Fridlyand for help with Bioconductor $R$, and Dr. R. Kramer for use of the WM115 melanoma cell line. This work was supported by Komen Foundation grant no. BCTRO0-000378 (F.M.W.).

\section{REFERENCES}

1.Hughes, T.R., M.J. Marton, A.R. Jones, C.J. Roberts, R. Stoughton, C.D. Armour, H.A. Bennett, E. Coffey, et al. 2000. Functional discovery via a compendium of expression profiles. Cell 102:109-126.

2.van 't Veer, L.J., H. Dai, M.J. van de Vijver, Y.D. He, A.A. Hart, M. Mao, H.L. Peterse, K. van der Kooy, et al. 2002. Gene expression profiling predicts clinical outcome of breast cancer. Nature 415:530-536.

3.Spellman, P.T., G. Sherlock, M.Q. Zhang, V.R. Iyer, K. Anders, M.B. Eisen, P.O. Brown, D. Botstein, et al. 1998. Comprehensive identification of cell cycle-regulated genes of the yeast Saccharomyces cerevisiae by microarray hybridization. Mol. Biol. Cell 12:3273-3297.

4.Hegde, P., R. Qi, K. Abernathy, C. Gay, S. Dharap, R. Gaspard, J.E. Hughes, E. Snesrud, et al. 2000. A concise guide to cDNA microarray analysis. BioTechniques 29:548-562.

5.Baugh, L.R., A.A. Hill, E.L. Brown, and C.P. Hunter. 2001. Quantitative analysis of mRNA amplification by in vitro transcription. Nucleic Acids Res. 29:e29.

6.Brazma, A. and J. Vilo. 2000. Gene expression data analysis. FEBS Lett. 480:17-24.

7.Relogio, A., C. Schwager, A. Richter, W. Ansorge, and J. Valcarcel. 2002. Optimization of oligonucleotide-based DNA microarrays. Nucleic Acids Res. 30:e51.

8.Hughes, T.R., M. Mao, A.R. Jones, J. Burchard, M.J. Marton, K.W. Shannon, S.M. Lefkowitz, M. Ziman, et al. 2001. Expres- sion profiling using microarrays fabricated by an ink-jet oligonucleotide synthesizer. Nat. Biotechnol.19:342-347.

9.Decraene, C., I. Reguigne-Arnould, C. Auffray, and G. Pietu. 1999. Reverse transcription in the presence of dideoxynucleotides to increase the sensitivity of expression monitoring with cDNA arrays. BioTechniques 27: 962-966.

10.Maniatis, T., E.F. Fritsch, and J. Sambrook. 1989. Molecular Cloning: A Laboratory Manual. CSH Laboratory Press, Cold Spring Harbor, NY.

11.Eisen, M.B., P.T. Spellman, P.O. Brown, and D. Botstein. 1998. Cluster analysis and display of genome-wide expression patterns. Proc. Natl. Acad. Sci. USA 95:14863-14868.

12.Ginzinger, D.G. 2002. Gene quantification using real-time quantitative PCR: an emerging technology hits the mainstream. Exp. Hematol. 30:503-551.

Received 27 May 2003; accepted 23 July 2003.
Address correspondence to:
Frederic M. Waldman
UCSF Comprehensive Cancer Center
2340 Sutter Street, Rm. 436
San Francisco, CA 94143-0808, USA
e-mail: waldman@cc.ucsf.edu 